\title{
$\dot{\nabla}_{\text {Nursing }}^{\text {Global Academic }}$
}

\section{Contribuição de um software para o registro, monitoramento e avaliação de feridas}

\author{
Contribution of software for recording, monitoring, and evaluating wounds
}

Contribución de software para el registro, seguimiento y evaluación de heridas

\author{
Rodrigo Madril Medeiros ${ }^{1}$ \\ ORCID: 0000-0002-8483-6133 \\ Márcio Neres dos Santos ${ }^{2}$ \\ ORCID: 0000-0002-4413-9878 \\ Vítor Monteiro Moraes ${ }^{3}$ \\ ORCID: 0000-0002-3540-7818 \\ Dagmar Elaine Kaiser ${ }^{4}$ \\ ORCID: 0000-0001-5652-4653 \\ Êrica Rosalba Mallmann Duarte ${ }^{4}$ \\ ORCID: 0000-0002-1792-327X \\ Karin Viegas ${ }^{5}$ \\ ORCID: 0000-0002-2546-9281
}

${ }^{1}$ Hospital de Clínicas de Porto Alegre. Rio Grande do Sul, Brasil.

2Pontifícia Universidade Católica do Rio Grande do Sul. Rio Grande do Sul, Brasil.

${ }^{3}$ Grupo Hospitalar Conceição. Rio Grande do Sul, Brasil.

${ }^{4}$ Universidade Federal do Rio Grande do Sul. Rio Grande do Sul, Brasil.

${ }^{5}$ Universidade Federal de Ciências da Saúde de Porto Alegre. Rio Grande do Sul, Brasil.

Como citar este artigo:

Medeiros RM, Santos MN, Moraes

VM, Duarte ERM, Viegas K.

Contribuição de um software para o registro, monitoramento e avaliação de feridas. Glob Acad Nurs.

2021;2(3):e146.

https://dx.doi.org/10.5935/2675-

5602.20200146

Autor correspondente:

Márcio Neres dos Santos

E-mail: nerespoa@gmail.com

Editor Chefe: Caroliny dos Santos Guimarães da Fonseca

Editor Executivo: Kátia dos Santos Armada de Oliveira

Submissão: 07-03-2021

Aprovação: 30-03-2021

\section{Resumo}

Objetivou-se descrever a construção de um software para registro, monitoramento e avaliação de feridas. Trata-se de uma pesquisa de produção tecnológica contendo o desenvolvimento de software. Foi concretizado em três etapas: gestão do projeto através da ferramenta Scrum, construção do software, validação de conteúdo e pré-teste. Como resultado aplicativo foi construído após revisão da literatura em bases de dados. $\mathrm{O}$ sistema de gestão permitiu cadastrar pacientes, realizar a avaliação e o monitoramento da evolução das lesões através de imagens e de outras ferramentas, tais como gráficos de resultados das escalas Braden Scale for Predicting Pressure Ulcer Risk e Pressure Ulcer Scale for Healing. Além disso, estão disponíveis informações sobre coberturas e alternativas para o tratamento. O desenvolvimento de um protótipo inicial permitiu a exploração das ideias, antes do investimento na produção, auxiliando na economia de tempo e recursos, modulando de acordo com as necessidades do público-alvo, o produto final a ser desenvolvido. Por conclusão, o presente estudo demonstrou que a construção do software atende à necessidade para o registro, monitoramento e a avaliação de feridas. As perspectivas são de que também possa ser utilizado para realização de simulação realística e treinamentos, além de possuir facilidade de acesso e manuseio.

Descritores: Enfermagem; Tecnologia; Feridas; Aplicativos Móveis; Software.

\section{Abstract}

The aim was to describe the construction of a software for recording, monitoring, and evaluating wounds. It is a technological production research containing software development. It was carried out in three stages: project management through the Scrum tool, software construction, content validation and pre-test. As a result, the app was built after reviewing the literature in databases. The management system allowed registering patients, performing the assessment, and monitoring of the evolution of the lesions through images and other tools, such as graphs of the results of the Braden Scale for Predicting Pressure Ulcer Risk and Pressure Ulcer Scale for Healing. In addition, information about coverage and treatment alternatives is available. The development of an initial prototype allowed the exploration of ideas, before investing in production, helping to save time and resources, modulating the final product to be developed according to the needs of the target audience. In conclusion, the present study demonstrated that the construction of the software meets the need for recording, monitoring, and evaluating wounds. The prospects are that it can also be used to carry out realistic simulation and training, in addition to having easy access and handling.

Descriptors: Nursing; Technology; Injuries; Mobile Applications; Software.

\section{Resumén}

El objetivo fue describir la construcción de un software para el registro, seguimiento y evaluación de heridas. Es una investigación de producción tecnológica que contiene desarrollo de software. Se llevó a cabo en tres etapas: gestión de proyectos a través de la herramienta Scrum, construcción de software, validación de contenido y pre-test. Como resultado, la aplicación se creó después de revisar la literatura en las bases de datos. El sistema de gestión permitió registrar a los pacientes, realizar la valoración y seguimiento de la evolución de las lesiones a través de imágenes y otras herramientas, como gráficas de los resultados de la Escala de Braden de Predicción del Riesgo de Úlceras por Presión y Escala de Curación de Úlceras por Presión. Además, se encuentra disponible información sobre la cobertura y las alternativas de tratamiento. El desarrollo de un prototipo inicial permitió la exploración de ideas, antes de invertir en producción, ayudando a ahorrar tiempo y recursos, modulando el producto final a desarrollar según las necesidades del público objetivo. En conclusión, el presente estudio demostró que la construcción del software responde a la necesidad de registrar, monitorear y evaluar las heridas. Las perspectivas son que también se puede utilizar para realizar simulaciones y entrenamientos realistas, además de tener un fácil acceso y manejo.

Descriptores: Enfermaría; Tecnología; Heridas; Aplicaciones Móviles; Software. 


\section{Introdução}

A construção de uma linha de cuidado e o financiamento para o cuidado com feridas é um desafio, especialmente, nos sistemas de saúde em que o tratamento não é reconhecido em sua magnitude, no sentido do impacto na qualidade de vida do paciente, adesão ao tratamento de outras doenças, redução do risco de infecções, redução nas taxas de permanência e (re)internações hospitalares.

Nos Estados Unidos da América (EUA) as feridas crônicas afetam cerca de 6,5 milhões de pacientes, sendo que os gastos anuais com o tratamento podem chegar a aproximadamente 25 bilhões de dólares ${ }^{1,2}$. Este fato também é percebido pelo lucro de bilhões de dólares pela indústria farmacêutica com os produtos de cuidados com feridas $^{3}$. Em países em desenvolvimento, assim como o Brasil, ainda existem outros desafios a serem vencidos, tais como: o reembolso aos procedimentos especializados no cuidado às feridas pelo sistema público de saúde e, também através da saúde suplementar. Assim como, o credenciamento dos profissionais de enfermagem aos planos de saúde privados e público e o uso de guidelines de melhores práticas de cuidado com feridas por parte dos profissionais.

Embora, não se tenha dados consolidados do número de pessoas que são afetadas por feridas crônicas ou agudas nos países em desenvolvimento, sabe-se que a prevalência e incidência é alta e provavelmente continue a aumentar. Assim como nos países desenvolvidos, esse fato se deve, em parte, pelo aumento da expectativa de vida da população, sendo agravado pelo aumento na prevalência da obesidade, diabetes e doenças cardiovasculares ${ }^{1,2,4}$. Os portadores de feridas, principalmente, as crônicas, sofrem com comorbidades como diabetes e hipertensão. De certa forma, estas patologias parecem ter, por si só, ofuscado o significado da ferida crônica como um problema de saúde.

A avaliação é uma das bases do tratamento de feridas, pois a partir dela serão tomadas as decisões acerca dos diferentes recursos (físicos, tecnológicos e humanos) a serem utilizados no contexto assistencial. A avaliação realizada pelo enfermeiro não é dada apenas daquilo que se vê, pois, o não visível pode levar a uma avaliação equivocada ou ser o motivo para retardar o processo de cicatrização ${ }^{5}$.

Para a avaliação de uma ferida é necessário o suporte da semiologia para a realização do exame clínico (anamnese e exame físico), que tem por finalidade auxiliar o enfermeiro na tomada de decisão quanto ao melhor tratamento disponível. Além disso, o exame clínico constitui a base para a inter-relação das demais etapas do processo de enfermagem ${ }^{5,6}$.

O desenvolvimento de ferramentas de suporte para o registro, avaliação e monitoramento é um passo importante para a abordagem sistematizada no tratamento das feridas. A incorporação de novas tecnologias acarreta novas demandas, em algumas situações, aumentando a intensidade do trabalho e fomentando o trabalho interdisciplinar 7,8 . A tecnologia está imbricada na inovação. Assim, a inovação com a geração de novas ideias ou a aplicação das ideias para uma nova situação, resulta na melhoria de um serviço, programa, estrutura, produtos e/ou processos ${ }^{7}$.

No contexto das tecnologias da informação, a utilização de softwares são recursos que vem se expandindo e tem se apresentado como área inovadora e com grande contribuição para o acesso de informações com eficácia e redução do tempo. Os sistemas de informação podem estar inseridos nas práxis do cuidado, auxiliando em todas as etapas do processo de enfermagem, contribuindo também para a formatação de indicadores gerenciais ${ }^{9}$.

Construir um software que contemple as práticas de enfermagem pode gerar impacto na qualificação do cuidado em diferentes âmbitos, pois as deficiências no processo assistencial aos pacientes com feridas estão fortemente ligadas à avaliação inadequada, registros falhos e a falta de monitoramento dessas feridas, entre outros $^{2,10,11}$. Assim, o presente estudo objetiva descrever a construção de um software para registro, monitoramento e avaliação de feridas.

\section{Metodologia}

Trata-se de uma pesquisa de produção tecnológica. É um tipo de investigação cujo objetivo é desenvolver um novo produto, programa ou modelo ${ }^{12,13}$. Foi realizado no período de abril a dezembro de 2018 , concretizado em três etapas: gestão do projeto através da ferramenta Scrum, construção do software, validação de conteúdo e pré-teste.

A ferramenta Scrum auxiliou na gestão da construção do software aplicativo, na possibilidade de verificar antecipadamente riscos de insucesso, além de tornar o processo mais ágil. Sua melhor vantagem foi a utilização de várias técnicas e processos já utilizados anteriormente e aprovadas de maneira organizada para o desenvolvimento do produto ${ }^{12}$.

A ferramenta Scrum sistematizou a gestão do projeto através da criação de uma lista contendo todas as funcionalidades desejadas para o produto, sendo que a lista não estava completa no início do projeto, pois a mesma foi atualizada de acordo com as necessidades do desenvolvimento do produto (Product Backlog); reuniões de trabalho (Sprint e Daily Meeting) visando delimitar o período de desenvolvimento de funcionalidades do produto que ocorreu em cada etapa concluída e entregue (Sprint-Sprint). Cada item definido para o Sprint foi distribuído na forma de tarefas (Quadro de Kanban) ${ }^{13}$. Estas tarefas foram distribuídas no organograma da Sprint, onde cada integrante da equipe assumiu e executou as tarefas de sua responsabilidade, uma após a outra até que todas fossem concluídas dentro do período combinado.

Além disso, foi realizado uma eleição pela equipe de modo a definir a pontuação de cada tarefa da Sprint, objetivando para os cumprimentos dos prazos (Planning Poker). Também foi adotado o Burndown, um gráfico utilizado para acompanhar o andamento da execução da Sprint. Ao final de cada Sprint eram realizadas reuniões de retrospectiva, conduzidas pelo Scrum Master (coordenador do projeto), em conjunto com os demais integrantes, cujo objetivo é identificar pontos positivos e negativos da Sprint $^{13}$. 
As principais tecnologias que foram utilizadas para o desenvolvimento do software foram as linguagens PHP (Hipertext Preprocessor), JavaScript e Bootstrap. O banco de dados escolhido foi o MySQL, que é um sistema de gerenciamento de banco de dados SQL Open Source desenvolvido e distribuído pela MySQL $A B$, que é o padrão mais comum usado para acessar banco de dados e é definido pelo Padrão n. ANSI/ISO SQL46.

A validação foi realizada por 18 expertises, sendo nove enfermeiros e nove profissionais de tecnologia da informação (TI). A seleção dos participantes iniciais e os indicados pelos seus pares obedeceu os seguintes critérios de seleção: possuir título de especialista em enfermagem dermatológica ou em enfermagem em estomaterapia ou ter conhecimento na área de prevenção e tratamento de feridas (enfermeiros); possuir graduação na área de tecnologia da informação e conhecimento em operacionalização de sistemas em relação à funcionalidade, confiabilidade e usabilidade de software e, também possuir experiência de no mínimo um ano na área de programação ou análise de sistemas (profissionais de $\mathrm{TI}$ ).

O número de participantes para cada grupo respeita a NBR ISO/IEC n.o 14598-648 (Norma Brasileira (International Organization for Standardization / International Electrotechnical Commission), que indica o mínimo de oito avaliadores para cada grupo para que se obtenha resultados confiáveis. A escolha dos participantes se deu pela metodologia snow ball (Bola de Neve), em que a identificação das expertises foi realizada pelo reconhecimento de seus pares, isto é, os participantes iniciais indicavam novos participantes, que por sua vez indicavam outros, e assim sucessivamente até que fosse alcançado o número recomendado. Deste modo, não é possível determinar a probabilidade de seleção de cada participante ${ }^{14}$.

Os avaliadores foram convidados por e-mail enviado pelo pesquisador, solicitando a participação no estudo. Esse e-mail também continha instruções: se a pessoa concordasse em participar, deveria acessar o link correspondente ao endereço do seu questionário e aceitar o Termo de Consentimento Livre e Esclarecido (TCLE). A mensagem ainda orientou que o link do software somente deveria ser acessado após a aceitação do termo, inserindo nome de usuário e senha fornecidos pelo pesquisador para cada avaliador.

Para guiar os avaliadores foi enviado um documento com orientações de acesso ao sistema, além de um tutorial, apresentado no próprio software, que continha um caso clínico fictício com todos os seus respectivos dados, necessários ao preenchimento do instrumento (exame físico, indicadores, prescrição de cuidados, entre outros). 0 preenchimento das questões da avaliação foi realizado pelos enfermeiros e profissionais de informática por meio de acesso ao link, utilizando-se o formulário Google ${ }^{\circledR}$. As respostas dos questionários foram recebidas automaticamente por essa ferramenta, a partir da conclusão da avaliação, formando um banco de dados para posterior análise.
A referência adotada neste projeto foi descrita na Norma ISO/IEC n.o 2501050, composto por oito características, que são subdivididas em subcaracterísticas, capazes de fornecer terminologia consistente para especificar, medir e avaliar sistemas e a qualidade dos produtos. Estes critérios de avaliação já foram testados em produtos semelhantes ${ }^{15,16}$. Foram recebidas todas as respostas aplicadas a cada característica e subcaracterística, conforme o julgamento dos avaliadores. A partir da obtenção dos dados, estes foram organizados e analisados em planilha do software Microsoft Excel ${ }^{\circledR}$.

Para a realização deste estudo, foram respeitados todos os preceitos éticos determinados pela Resolução $\mathrm{n}$. 466, de 12 de dezembro de 2012, do Conselho Nacional de Saúde ${ }^{17}$. Este projeto de pesquisa foi submetido e aprovado pelo Comitê de Ética em Pesquisa da Universidade Federal de Ciências da Saúde de Porto Alegre, número do Parecer CEP/UFCSPA n. 1. 500. 677 e CAAE: 54748216.3.0000.5345.

O aplicativo está em processo de registro no INPE.

\section{Resultados}

O software Wounds Monitoring visa ser uma ferramenta de trabalho para os enfermeiros ou outros profissionais da saúde, cuja finalidade é monitorar as lesões de pele. Neste sistema de gestão é possível cadastrar pacientes, realizar a avaliação e o monitoramento da evolução das lesões através de imagens e de outras ferramentas, tais como gráficos de resultados das Escalas de Braden (Braden Scale for Predicting Pressure Ulcer Risk) e PUSH (Pressure Ulcer Scale for Healing). Além disso, estão disponíveis informações sobre coberturas e alternativas para o tratamento.

O desenvolvimento de um protótipo inicial permitiu a exploração das ideias, antes do investimento na produção, auxiliando na economia de tempo e recursos, modulando de acordo com as necessidades do público-alvo o produto final a ser desenvolvido.

$\mathrm{Na}$ área de desenvolvimento de software os protótipos são reconhecidos por sua fidelidade, podendo ser classificado em baixa, média e alta fidelidade. Sendo que a alta fidelidade é o mais aproximado do produto final, por executar a maior parte das funções desejadas ${ }^{18}$. O software Wounds Monitoring está enquadrado como de alta fidelidade, pois, além de ser altamente funcional, se aproxima do produto final a ser desenvolvido.

Para colocar o Wounds Monitoring em funcionamento, foi necessário fazer o registro de um domínio e, posteriormente, firmar contrato com um servidor para realizar a hospedagem do sistema. Cumpridas essas etapas, foi possível disponibilizar o protótipo para acesso via $W e b$, de qualquer lugar e a qualquer hora. Para que isto aconteça e para realizar a avaliação, o profissional deve possuir um aparelho com acesso à internet e ter nome de usuário e senha de acesso, posteriormente a seu aceite (TCLE).

O software foi desenvolvido em formato modular, em concomitância com as etapas do processo de avaliação de feridas e outras lesões de pele. Os módulos são assim 
denominados: Pacientes, Guia de Produtos, Assistência à Saúde, Tutorial, Ajuda e Sobre nós.

Ao digitar o domínio, o usuário é direcionado à primeira tela, denominada tela inicial do Wounds Monitoring, que serve para realizar o login de acesso ao
Medeiros RM, Santos MN, Moraes VM, Duarte ERM, Viegas K sistema. A segunda tela tem como função dar as boas-vindas aos usuários do Wounds Monitoring e acesso ao prontuário dos pacientes cadastrados e, também, a possibilidade de cadastrar um novo paciente (Figura 1).

Figura 1. Tela de boas-vindas do Wounds Monitoring e acesso ao Prontuário dos pacientes cadastrados a e ao ícone para cadastrar um novo paciente. Porto Alegre, RS, Brasil, 2021

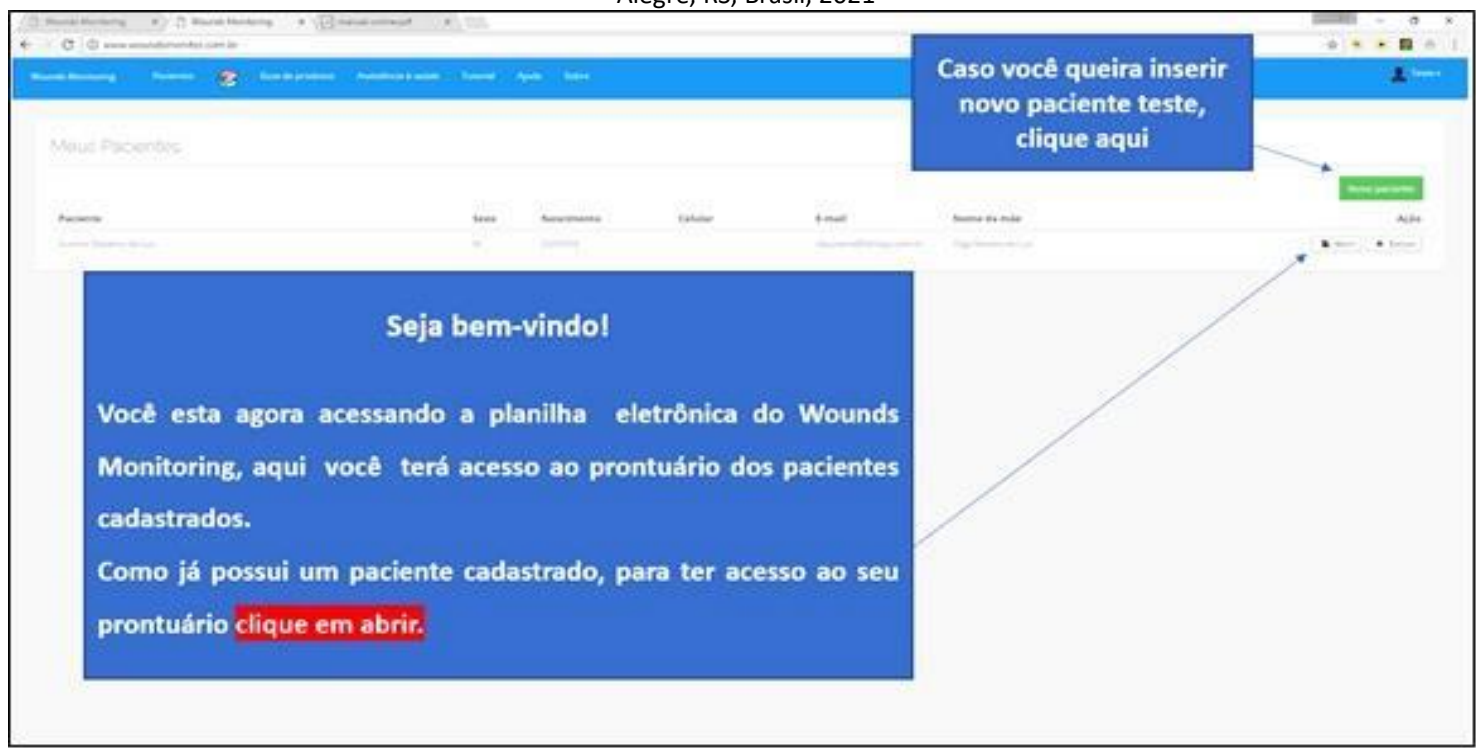

Fonte: Wounds Monitor

Além disso, como mostrado na Figura 2, estão disponíveis na barra superior desta tela os ícones:

Sobre que tem por função a apresentar de forma clara e objetiva o que é e qual a função do software;

Ajuda que tem por função ser um canal de comunicação direta com o desenvolvedor para esclarecer dúvidas e enviar sugestões e críticas;

Tutorial que apresenta o passo a passo do funcionamento do Wounds Monitoring;

Assistência à saúde que na versão protótipo está indisponível, porém futuramente, na versão final, disponibilizará dicas de serviços, tais como farmácias, profissionais, laboratórios, entre outros;

Guia de produtos que apresenta de forma sintetizada algumas sugestões de produtos para o tratamento de feridas disponíveis no mercado; e

Utilidades que disponibiliza algumas ferramentas de classificação de feridas, índices prognósticos, escalas preditoras, entre outros.

Figura 2. Tela de acesso ao Prontuário dos pacientes cadastrados e ao ícone para cadastrar um novo paciente. Barra de ferramentas. Porto Alegre, RS, Brasil, 2021

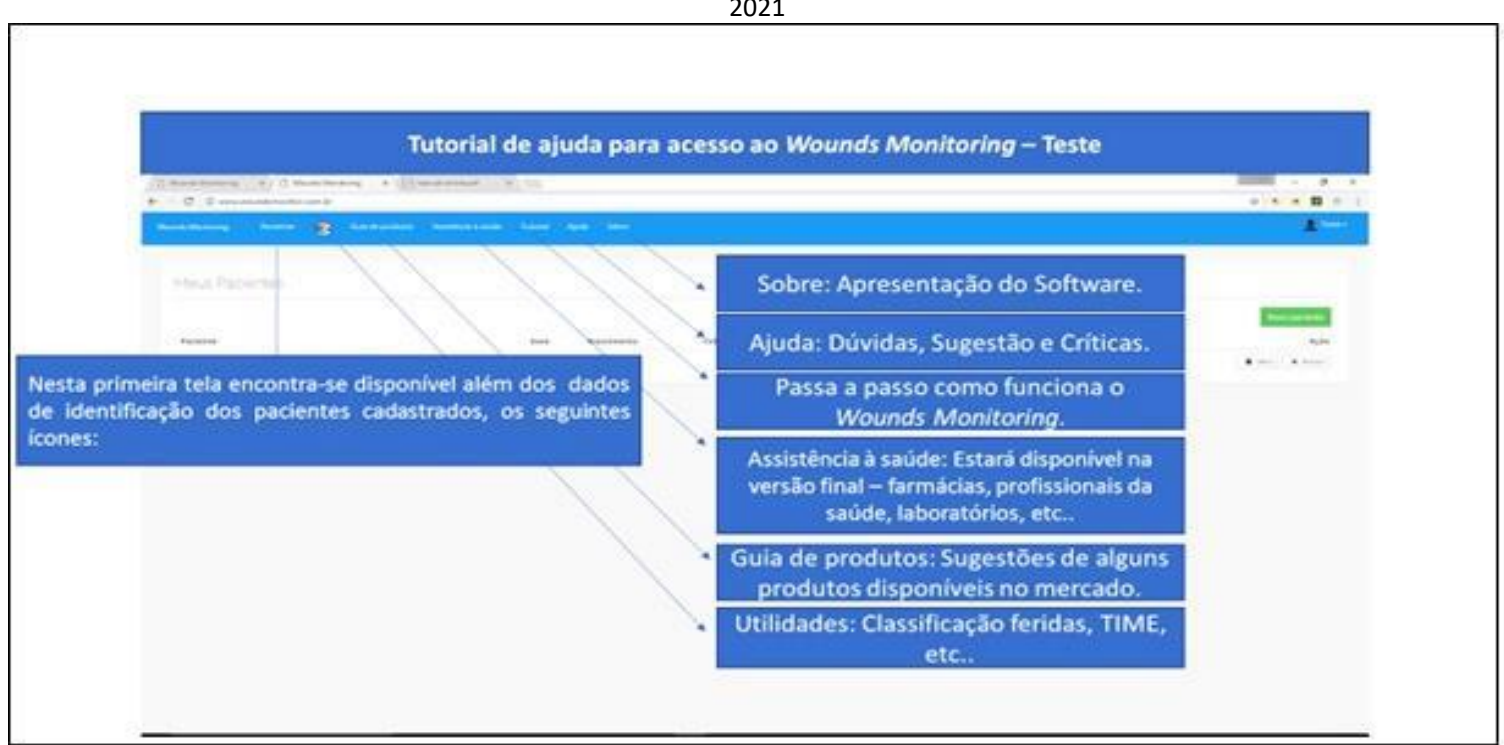

Fonte: Wounds Monitor. 
Construção de um software para o registro, monitoramento e avaliação de feridas Medeiros RM, Santos MN, Moraes VM, Duarte ERM, Viegas K

Ao clicar em "novo paciente", o sistema é direcionado para a tela de cadastramento e da anamnese do paciente (Figura 3). Após o cadastramento do paciente, o usuário é direcionado para a tela de seus respectivos tratamentos. O módulo é composto por três abas que devem ser preenchidas com as informações obtidas através da realização de exame físico e do anexo de imagens da ferida para o monitoramento. Nesta tela, o usuário encontra informações referentes aos tratamentos e localização da ferida. Caso seja necessário abrir um novo tratamento para este paciente, deverá ser clicado o ícone "novo tratamento" (Figura 4).

Figura 3. Tela para cadastrar um novo paciente, anamnese. Porto Alegre, RS, Brasil, 2021

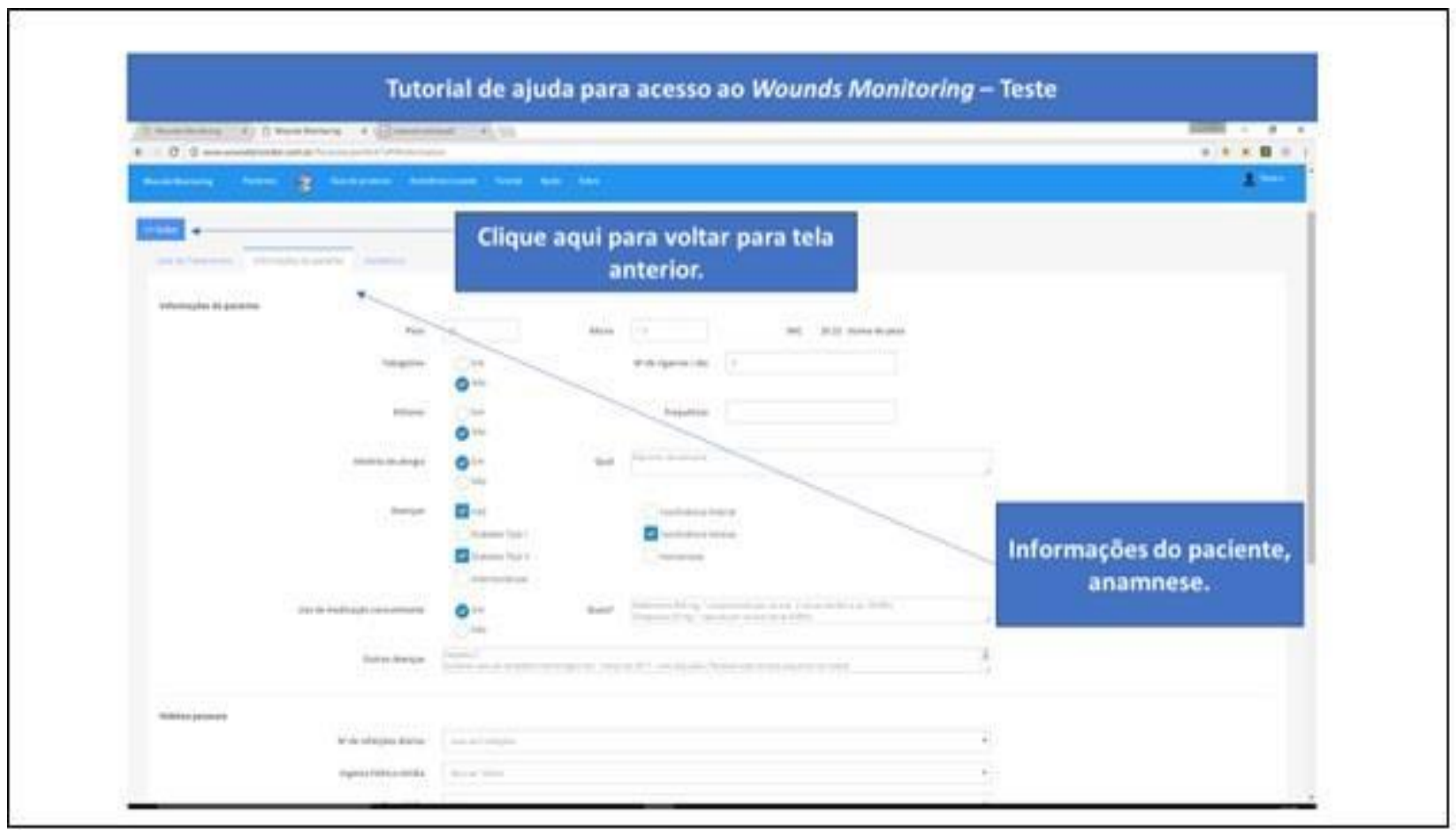

Fonte: Wounds Monitor.

Figura 4. Tela para inserir um novo tratamento no paciente selecionado. Porto Alegre, RS, Brasil, 2021

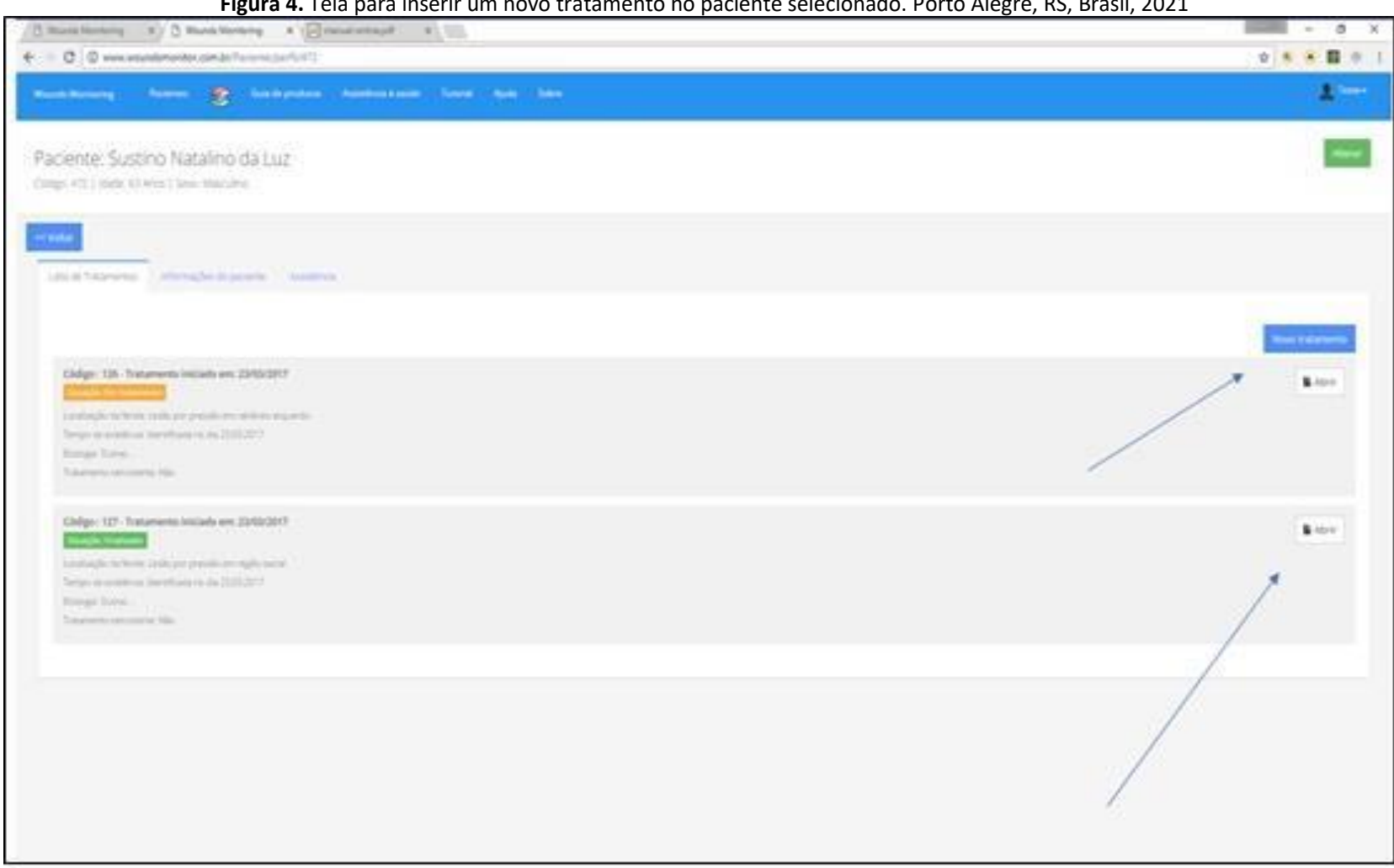

Fonte: Wounds Monitor 
Na tela tratamento é oferecido ao usuário de forma resumida, o monitoramento das intervenções, a evolução e as imagens das lesões (Figuras 5).

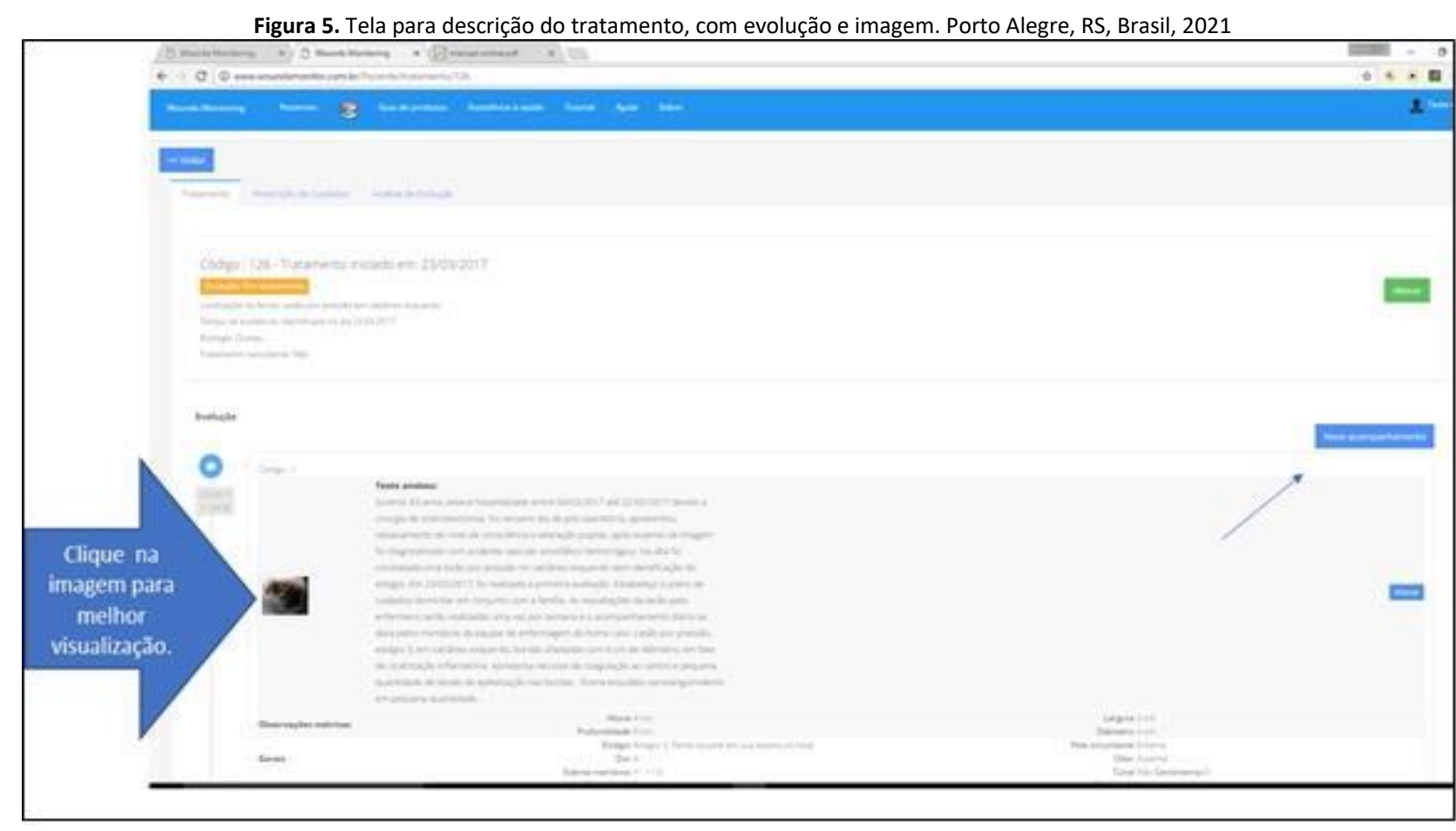

Fonte: Wounds Monitor.

Na tela apresentada na Figura 6 pode ser conferido o acompanhamento das lesões conforme ordem cronológica da avaliação. Isto facilita a evolução de cada lesão.

Figura 6. Tela resumo dos tratamentos propostos, com imagem da lesão. Porto Alegre, RS, Brasil, 2021

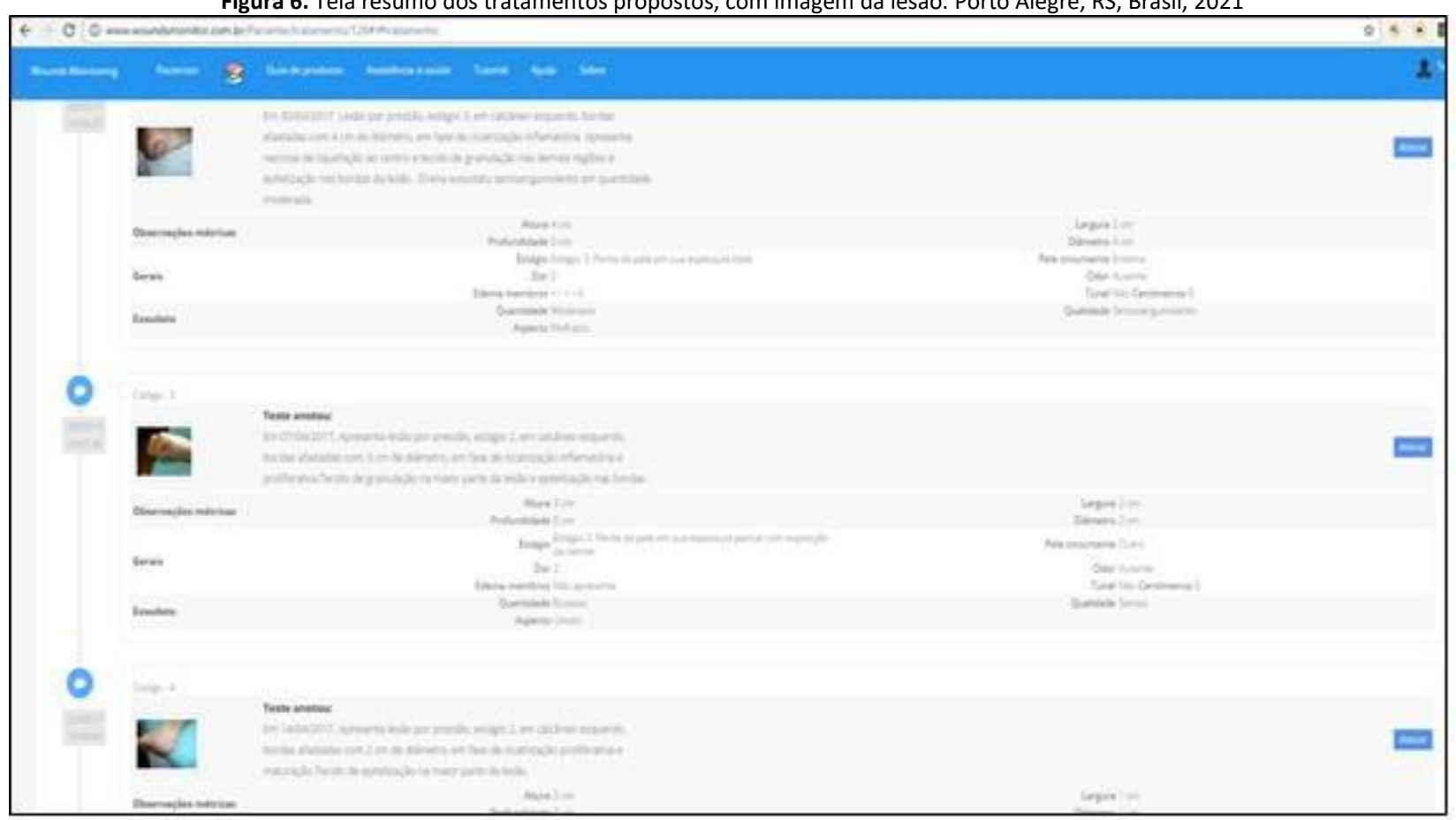

Fonte: Wounds Monitor.

$\mathrm{Na}$ tela apresentada na Figura 7, de acompanhamento, o usuário deve preencher as observações métricas, conforme sua avaliação, que possibilitarão gerar o gráfico de monitoramento do PUSH. Através do gráfico gerado, o usuário pode monitorar em tempo real as escalas de PUSH e Braden do paciente (Figura 8). 


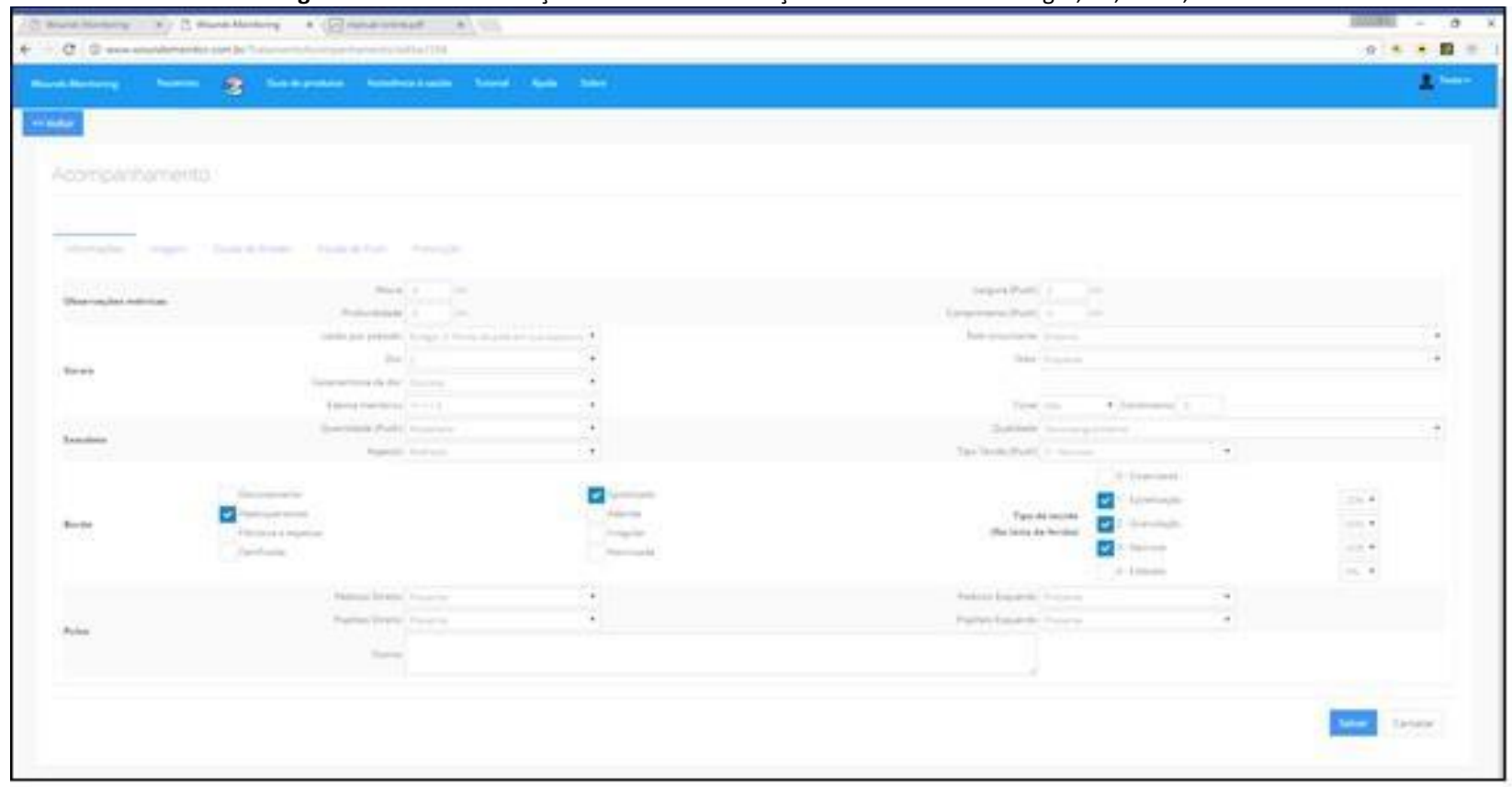

Fonte: Wounds Monitor.

Figura 8. Gráfico da Escala de PUSH gerado pelas observações métricas realizadas. Porto Alegre, RS, Brasil, 2021

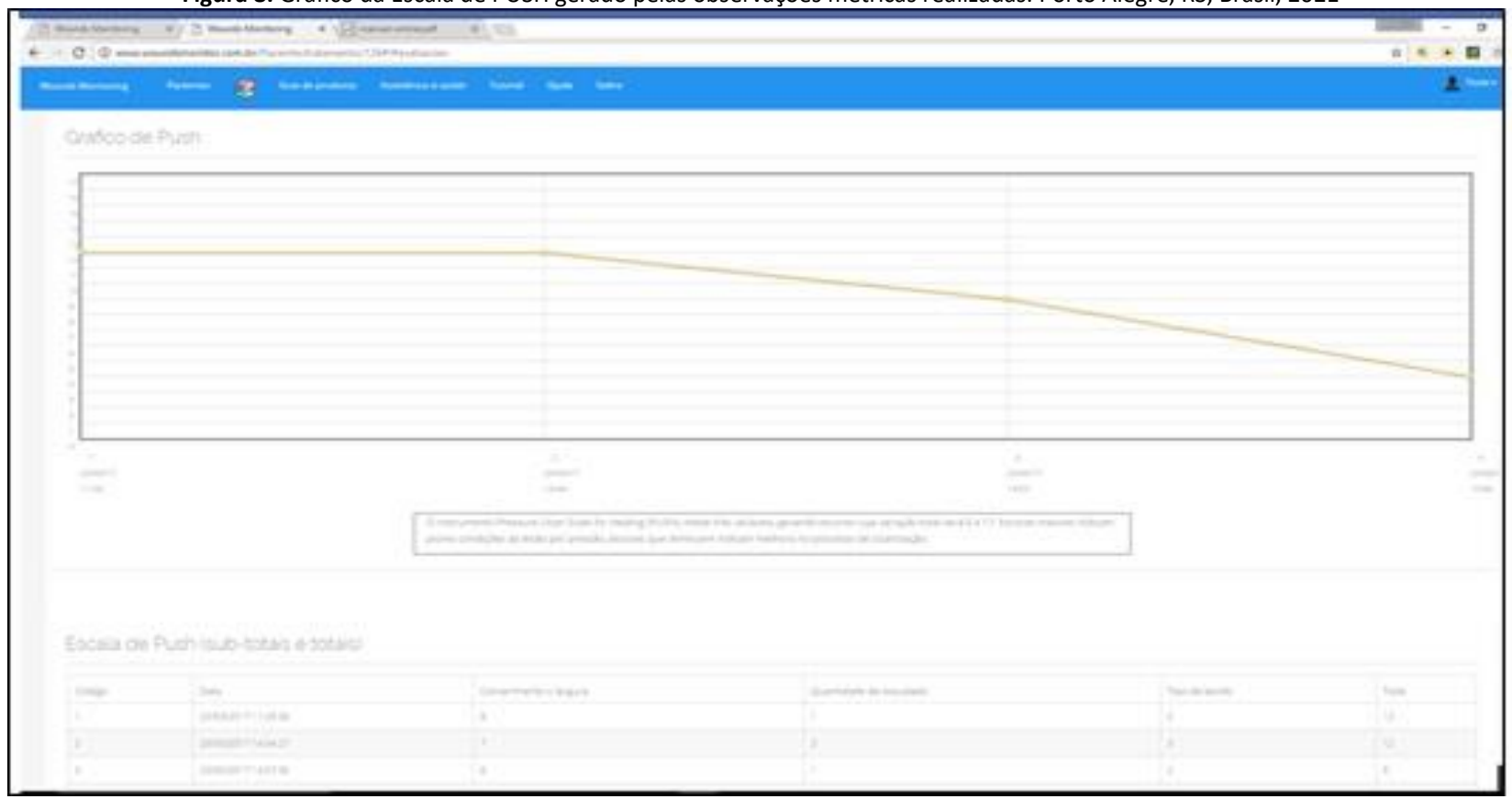

Fonte: Wounds Monitor

Sendo os registros de enfermagem da responsabilidade de técnicos, auxiliares de enfermagem e enfermeiros, a evolução de enfermagem é uma atribuição privativa do enfermeiro, além de se constituir em um dever, de acordo com o Código de Ética e demais legislações pertinentes ${ }^{19}$.

A responsabilidade do profissional poderá ocorrer no âmbito ético, legal, administrativo, cível e criminal. Em função deste aspecto, está previsto na tela de acompanhamento, um espaço específico para o registro de enfermagem sobre a lesão e a evolução de enfermagem, assim como um campo para arquivar as imagens, tendo um monitoramento visual da ferida (Figura 9).
A tela prescrição do Wounds Monitoring oferece a possibilidade de escolher o tipo de produto a ser utilizado no tratamento da ferida, bem como a prescrição dos cuidados. Todos os dados descritos e marcados são selecionados para a elaboração da prescrição de enfermagem.

Os produtos são estabelecidos de acordo com a avaliação do usuário, considerando os tipos de tecidos e de exsudato identificados na lesão. Também é apresentada a indicação do tempo de troca para cada tipo de curativo. Ao clicar em "salvar", os dados são armazenados automaticamente no servidor, podendo ser acessados através do software (Figura 10). 
Construção de um software para o registro, monitoramento e avaliação de feridas Medeiros RM, Santos MN, Moraes VM, Duarte ERM, Viegas K Figura 9. Acompanhamento com descrição da evolução de enfermagem. Porto Alegre, RS, Brasil, 2021

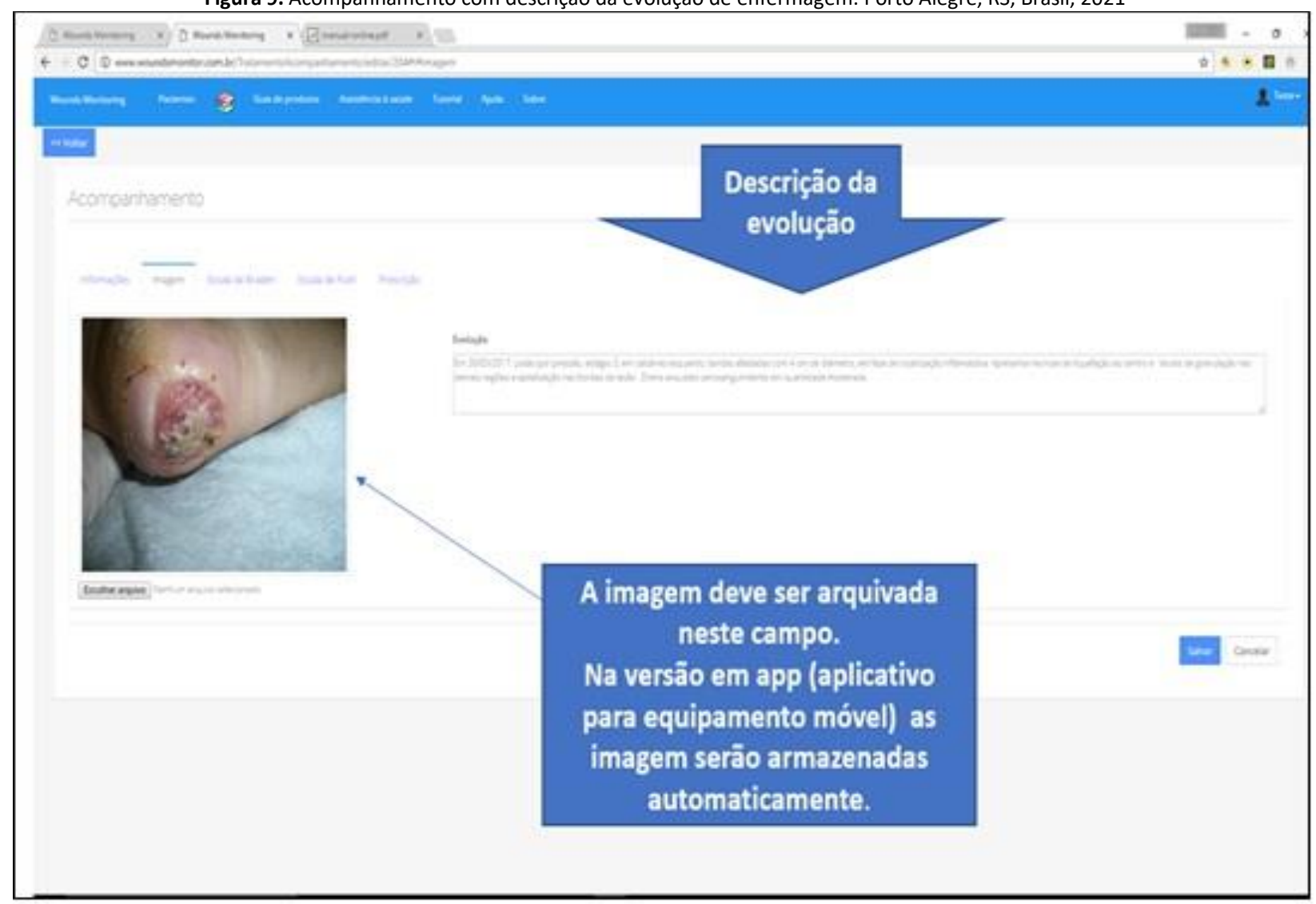

Fonte: Wounds Monitor.

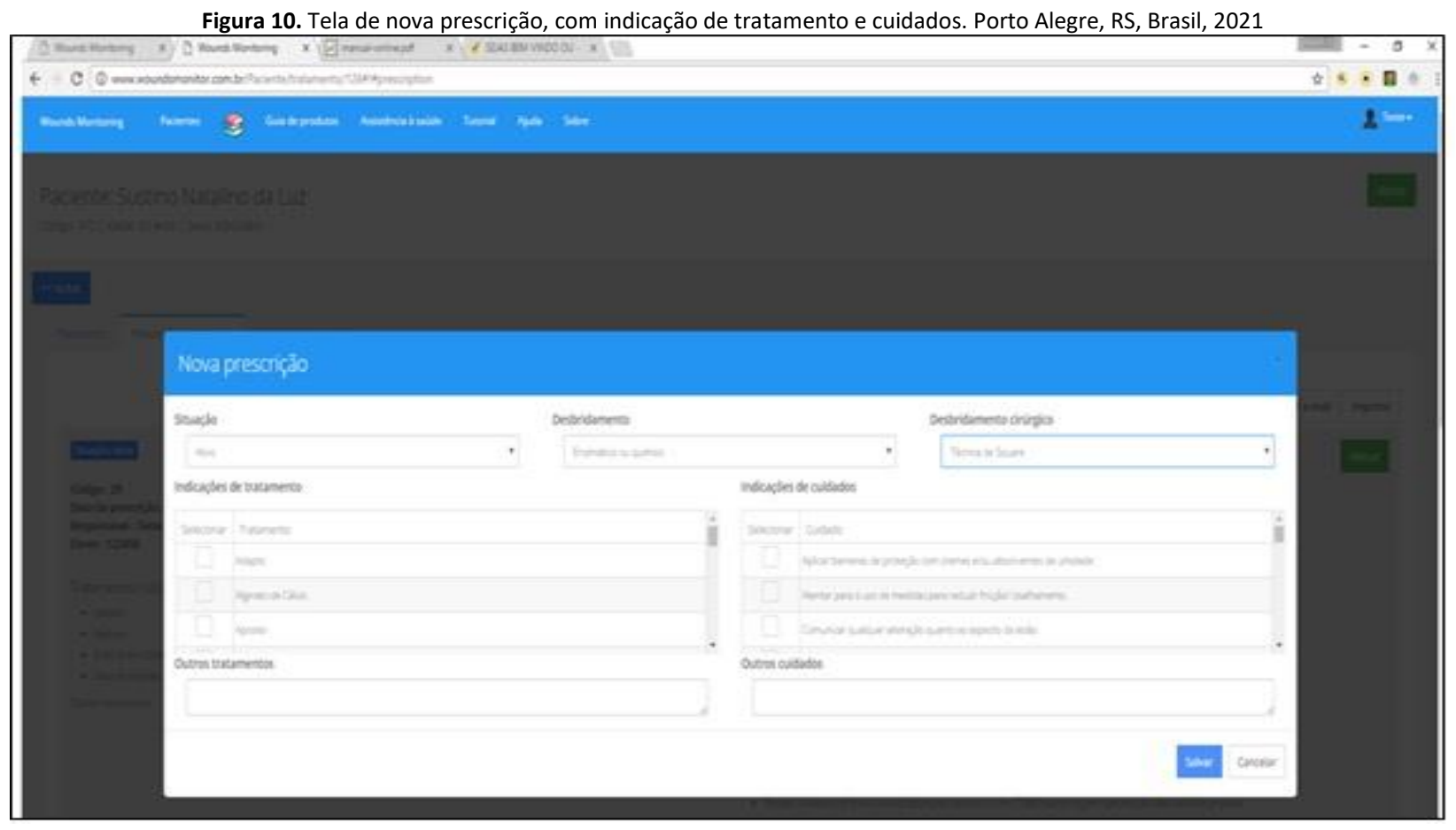

Fonte: Wounds Monitor.

O Wounds Monitoring gera uma prescrição que pode ser impressa ou enviada por e-mail para os profissionais habilitados no sistema. Os itens gerados para a impressão são: Identificação do paciente; Localização da ferida; Evolução; Prescrição de cuidados; Tratamento indicado; e Gráficos de monitoramento de Braden e PUSH.
Esta prescrição poderá ser impressa por qualquer profissional, entretanto o sistema apresenta um bloqueio permitindo unicamente que a mesma seja modificada pelo enfermeiro ou médico habilitados no sistema. O relatório final de acompanhamento do Wounds Monitoring para cada paciente avaliado poderá ser impresso (Figura 11). 


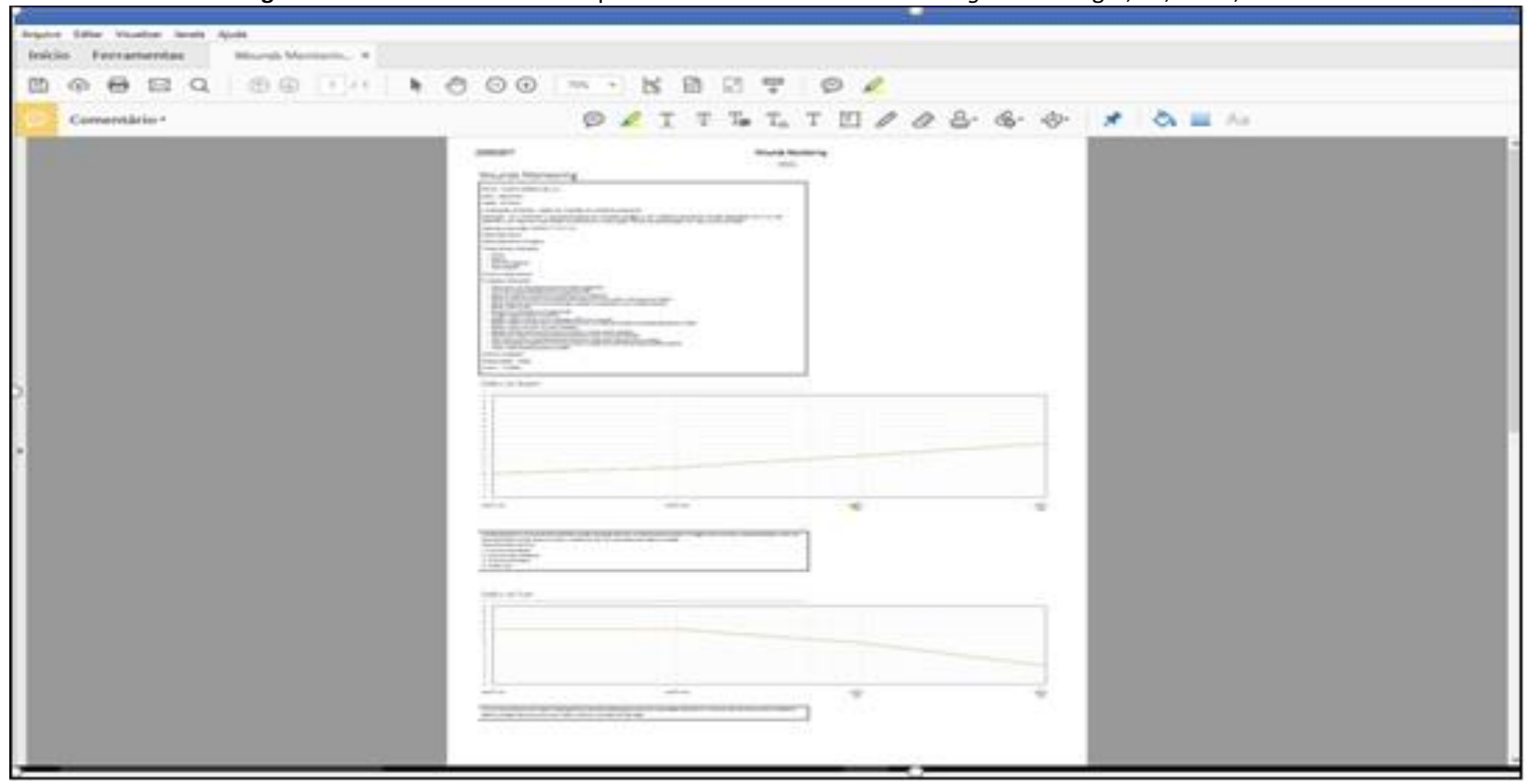

Fonte: Wounds Monitor.

\section{Discussão}

As diretrizes, protocolos, cartilhas e algoritmos seguem como os principais fios condutores dos enfermeiros no desenvolvimento de maior acurácia na avaliação das feridas crônicas e agudas. De acordo com a legislação vigente, cabe ao enfermeiro coordenar a equipe de enfermagem, conduzir e viabilizar a qualidade do cuidado e direito do paciente de forma digna, segura, legal e ética ${ }^{20}$.

O papel do enfermeiro na área dermatológica está vinculado a um arsenal tecnológico específico, exigindo conhecimentos e habilidades relacionados ao manuseio de materiais e equipamentos e às necessidades dos pacientes que dependem delas. O enfermeiro no cuidado às feridas desempenha cinco funções essenciais no contexto das práticas em saúde: cuidar, educar, coordenar, colaborar e supervisionar. Tais funções são desenvolvidas de forma integrada e concomitante. Contudo, ora são mais centradas em uma, ora em outra, ora em todas elas, ou seja, não se dissociam.

A construção do software Wounds Monitoring surgiu a partir de reflexões sobre as dificuldades que os profissionais têm em sistematizar as ações no cuidado com feridas, assim como no monitoramento efetivo dessas ações. No caso de pacientes com feridas, os indicadores de qualidade assistencial podem ser elevados a partir da sistematização da assistência prestada, visto que na maior parte das vezes os cuidados com feridas (troca de curativos, evolução das lesões, intercorrências) não são registrados adequadamente ${ }^{11,19}$.

Os softwares são ferramentas de suporte assistencial e, não tem a pretensão de substituir o pensamento crítico e o raciocínio clínico do profissional. É necessário reforçar que a documentação adequada garante o sucesso do tratamento e acompanhamento evolutivo da ferida. Nesse contexto, Wounds Monitoring é uma tecnologia em saúde a ser utilizada pelos enfermeiros nos diferentes contextos assistenciais que acolham pacientes com feridas.

Existe a perspectiva que o software possa ser utilizado na atenção primária à saúde e no âmbito hospitalar, visto que oferece a possibilidade de ser consultado em dispositivos móveis e futuramente na modalidade off-line. A ferramenta foi elaborada a partir de um banco de dados composto de dois cadastros. Semelhante ao dispositivo construído por estudo ${ }^{21}$, o primeiro ou principal é o cadastro do paciente que contém seus dados pessoais, bem como a avaliação do profissional em relação aos aspectos clínicos do paciente. O segundo, vinculado ao primeiro, é o cadastro da avaliação da lesão, onde é feito o registro da avaliação (tipo de ferida, mensuração da área da ferida em $\mathrm{cm}^{2}$, tipo e quantidade do exsudato, tipo de tecido, e a localização anatômica onde a lesão se encontra.

O desenvolvimento do software foi embasado na literatura e nas melhores evidências clínicas, o que pode fornecer maiores subsídios técnicos e clínicos. Assim como, quanto ao processo de validação do protótipo, foram incluídas as contribuições das expertises, que forneceram informações relevantes para modificação da escrita e, até mesmo, da apresentação gráfica. Atingindo o percentual de $89 \%$ das expertises, concordaram com a aplicabilidade do software na prática clínica, ou seja, consideram uma ferramenta com potencial relevante capaz de apoiar a decisão do profissional na avaliação, monitoramento e na escolha do tratamento da ferida.

Nas últimas décadas vários métodos padronizados foram desenvolvidos para permitir aos profissionais monitorarem as lesões e, consequentemente, avaliar o efeito de uma intervenção. Instrumentos de avaliação melhoram e estimulam a comunicação entre os profissionais da equipe de saúde e possibilitam atingir os objetivos esperados com maior brevidade ${ }^{11}$. Entretanto, o que se 
observa são instrumentos desenvolvidos para avaliação de úlceras por pressão e não de feridas crônicas.

Um estudo de revisão integrativa sinalizou que diferentes sistemas de classificação foram desenvolvidos para avaliar as lesões por pressão, utilizando o estadiamento como, por exemplo, o sistema EPUAP (European Pressure Ulcer Advisory Panel). Outras ferramentas foram desenvolvidas para avaliar a cicatrização das feridas, tais como o PSST (Pressure Score Status Tool) e o PUSH (Pressure Ulcer Scale for Healing), ambas adaptadas transculturalmente para a língua portuguesa, entre outras escalas e escores já publicados internacionalmente. O objetivo dessas ferramentas é proporcionar aos profissionais informações que demostram se o tratamento foi efetivo e se a evolução da lesão está ocorrendo de forma adequada. 0 Wounds Monitoring foi elaborado e validado, levando em consideração os itens mínimos e necessários para avaliar uma ferida (mensuração da ferida, tipo de tecido, exsudato, quantidade de exsudato sinais de inflamação e/ou infecção, entre outros), utilizando escalas já validadas ${ }^{22-24}$.

Além disso, o software permite ao usuário escolher diferentes tipos de cobertura que podem ser utilizadas no tratamento. A maioria das avaliações feitas pelas expertises superaram as expectativas iniciais do estudo, chegando a percentuais acima de $94 \%$ de aprovação. O software foi avaliado como uma ferramenta capaz de ajudar o profissional de saúde na avaliação e monitoramento de feridas, bem como na escolha da cobertura adequada.

A cobertura pode ser definida como um meio terapêutico que consiste na limpeza e aplicação de material sobre uma ferida para sua proteção, absorção e drenagem, com o intuito de melhorar as condições do leito da ferida e auxiliar em sua resolução. Curativos podem ser, em algumas ocasiões, o próprio tratamento definitivo; em outras, apenas uma etapa intermediária para o tratamento cirúrgico ${ }^{25}$. No software são apresentadas algumas possibilidades de coberturas, sejam os curativos passivos (inertes) e aqueles com princípios ativos. Atualmente, existe no mercado uma variedade de coberturas e outros dispositivos de tratamento para feridas. Entretanto, cabe aos profissionais da saúde fazer a melhor escolha, sem nunca esquecer o quadro sistêmico que está envolvido no tratamento de uma ferida ${ }^{25}$.

Cabe ressaltar que o software facilitou o registro sistematizado da ferida, sua evolução e monitoramento. A partir dos registros no Wounds Monitoring foi possível identificar o tratamento em curso e os alguns fatores que poderiam interferir no processo de cicatrização. Registros inadequados em prontuários deixam a assistência mais suscetível a um evento adverso e comprometer a segurança do paciente ${ }^{26,27}$. O software torna-se um meio que pode auxiliar na garantia da comunicação efetiva.

\section{Conclusão}

Tecnologias como Wounds Monitoring fortalecem a cultura do uso de evidência científica no processo de tomada de decisão, influenciando conceitos e linguagens adotadas em deliberações clínicas por parte dos profissionais de saúde. A partir das proposições tecnológicas realizadas por esse estudo possibilitará aos enfermeiros acesso à informação, bem como permitirá a formação de um banco de dados. Além das possibilidades e benefícios aos profissionais já discutidos ao longo do presente estudo, é importante enfatizar que a ferramenta possibilita avaliação e monitoramento de feridas de maneira ágil e sistematizada, por meio de um sistema com interface amigável e autoexplicativa, com possibilidade de ter baixo custo.

O presente estudo demonstrou que a construção do software atende à necessidade para o registro, monitoramento e a avaliação de feridas. As perspectivas são de que também possa ser utilizado para realização de simulação realística e treinamentos, além de possuir facilidade de acesso e manuseio.

\section{Referências}

1. Sen CK, Gordillo GM, Roy S, Kirsner R, Lambert L, Hunt TK, et al. Human skin wounds: a major and snowballing threat to public health and the economy. Wound Repair Regen. 2010;17(6):763-71.

2. Crovetti G, Martinelli G, Issi M, Barone M, Guizzardi M, Campanati B, et al. Platelet gel for healing cutaneous chronic wounds. Transfus Apher Sci. 2004;30(2):145-51. DOI: 10.1016/j.transci.2004.01.004

3. Agency for Healthcare Research and Quality. What are the best practices in pressure ulcer prevention that we want to use? Agency for Healthcare Research and Quality [Internet]. 2014 [acesso em 04 jun 2020]. Disponível em: http://www.ahrq.gov/professionals/systems/hospital/pressureulcertoolkit/putool3.html

4. Hjort A, Gottrup F. Cost of wound treatment to increase significantly in Denmark over the next decade. J Wound Care. 2010;19(5):173-84. DOI: 1.12968/jowc.2010.19.5.48046

5. Prazeres SJ. Tratamento de feridas: teoria e prática. Porto Alegre: Moriá; 2009.

6. Rabelo ER, Aliti GB. Exame Físico. In: SOUZA, EM. Casos clínicos para a enfermagem. Porto Alegre: Moriá; 2010.

7. Souza ML, Prado ML, Dal Sasso GTM, et al. A inovação tecnológica e o cuidado de enfermagem. Temperamentvm [Internet]. 2010 [acesso em 04 jun 2020];11. Disponível em: /temperamentum/tn11/t7172p.php

8. Salvador PTCO, Oliveira RKM, Costa TD, et al. Tecnologia e inovação para o cuidado. Rev. enferm. UERJ [Internet]. 2012 [acesso em 04 jun 2020];20(1):111-7. Disponível em: https://www.e-publicacoes.uerj.br/index.php/enfermagemuerj/article/view/4004/2773

9. Lima DFB, Braga ALS, Fernandes JL, Brandão ES. Sistema de informação em saúde: conceções e perspectivas dos enfermeiros sobre o prontuário eletrônico do paciente. Rev. Enf. Ref [Internet]. 2011 [acesso em 04 jun 2020];3(5):113-19. Disponível em: http://www.scielo.mec.pt/scielo. php?script=sci_arttext\&pid=S0874-02832011000300012\&lng=pt\&nrm=iso 
10. World Union of Wound Healing Societies (WUWHS). Principles of best practice: Diagnostics and wounds. A consensus document. London: MEP Ltd; 2008.

11. Cowan T. Wound Care Handbook 2014-2015. 7. ed. London: MA Healthcare; 2015.

12. Rocha PK, Prado ML, Wal ML, Carraro TE. Cuidado e tecnologia: aproximações através do Modelo de Cuidado. Rev Bras Enferm [Internet]. 2008 [acesso em 04 jun 2020];61(1):113-6. Disponível em: http://www.scielo.br/pdf/reben/v61n1/18.pdf

13. Kniberg H, Skarin M. Kanban e scrum obtendo o melhor de ambos. Estados Unidos: C4Media; 2009.

14. Biernacki P, Waldorf D. Snowball Sampling: Problems and techniques of Chain Referral Sampling. Sociological Methods \& Research. 1981;2:141-63.

15. Oliveira NB. Avaliação de qualidade do registro eletrônico do processo de enfermagem. 2012. Tese (Doutorado) - Universidade de São Paulo, São Paulo, 2012.

16. Sperandio DJ. A tecnologia computacional móvel na Sistematização da assistência de enfermagem: avaliação de um software-protótipo. 2008. Tese (Doutorado) - Escola de Enfermagem, Universidade de São Paulo, Ribeirão Preto, 2008.

17. Ministério da Saúde (BR). Conselho Nacional de Saúde. Resolução n.o 466, de 12 de dezembro de 2012. Aprova as diretrizes e normas regulamentadoras de pesquisas envolvendo seres humanos [Internet]. Brasília (DF): MS; 2012 [acesso em 04 jun 2020]. Disponível em: https://bvsms.saude.gov.br/bvs/saudelegis/cns/2013/res0466_12_12_2012.html

18. Pressmann RS. Engenharia de Software. São Paulo: McGraw Hill; 2011.

19. Conselho Federal de Enfermagem (COFEN). Guia de recomendações para registro de enfermagem no prontuário do paciente e outros documentos de enfermagem. Brasília (DF): COFEN; 2016.

20. Melo AF. Elaboração e validação de um instrumento de registro de feridas crônicas. Uberaba. Dissertação [Mestrado em Enfermagem] Universidade Federal do Triângulo Mineiro; 2015.

21. Cunha DRD, Dutra RAA, Salomé GM, Ferreira LM. Construção de um aplicativo multimídia em plataforma móvel para tratamento de feridas com laserterapia. Rev. enferm. UFPE on line [Internet]. 2018 [acesso em 20 jun 2020]; 1241-1249. Disponível em: https://periodicos.ufpe.br/revistas/revistaenfermagem/article/viewFile/230676/28868

22. National Pressure Ulcer Advisory Panel. European Pressure Ulcer Advisory Panel and Pan Pacific Pressure Injury Alliance. Prevention and Treatment of Pressure Ulcers: Quick Reference Guide. Emily Haesler (Ed.). Osborne Park: Cambridge Media; 2014.

23. Alves DFS, Almeida AO, Silva JLG, Morais FI, Dantas SRPE, Alexandre NMC. Translation and adaptation of bates-jensen wound assessment tool for Brazilian culture. Texto Contexto Enferm. 2015 jul-set;24(3):826-33. https://doi.org/10.1590/0104-07072015001990014

24. Aron S, Gamba MA. Preparo do Leito da Ferida e a História do TIME. Estima [Internet]. 2010 [acesso em 04 jun 2020];7(1):20-24. Disponível em: https://www.revistaestima.com.br/index.php/estima/article/view/264

25. Smaniotto PHDS, Ferreira MC, Isaac C, Galli R. Sistematização de curativos para o tratamento clínico das feridas. Rev. Bras. Cir. Plást. 2012;623-626. https://doi.org/10.1590/S1983-51752012000400026

26. Agência Nacional de Vigilância Sanitária (ANVISA). Assistência segura: uma reflexão teórica aplicada à prática [Internet]. 1. ed. Brasília (DF): 2013 [acesso em 20 jun 2020]. Disponível em: www20.anvisa.gov.br/segurançadopaciente/imagens

27. Santos MAQF, Santos CM, Silva ALNV, Orlandi TS, Oliveira LD. Auditoria de enfermagem: aspectos da qualidade da assistência e do seu registro no prontuário. Glob Acad Nurs. 2020;1(2):e33. https://dx.doi.org/10.5935/2675- 5602.20200033 